\title{
Femtosecond photon-counting receiver
}

\author{
Michael A. Krainak*a, Timothy M. Rambo ${ }^{\text {, }}$ \\ Guangning Yang ${ }^{\mathrm{a}}$, Wei Lu ${ }^{\mathrm{a}}$, Kenji Numata ${ }^{\mathrm{a}}$ \\ aNASA Goddard Space Flight Center, Greenbelt, MD USA 20771; \\ borthwestern University, Evanston, Illinois, USA
}

\begin{abstract}
An optical correlation receiver is described that provides ultra-precise distance and/or time/pulsewidth measurements even for weak (single photons) and short (femtosecond) optical signals. A new type of optical correlation receiver uses a fourthorder (intensity) interferometer to provide micron distance measurements even for weak (single photons) and short (femtosecond) optical signals. The optical correlator uses a low-noise-integrating detector that can resolve photon number. The correlation (range as a function of path delay) is calculated from the variance of the photon number of the difference of the optical signals on the two detectors. Our preliminary proof-of principle data (using a short-pulse diode laser transmitter) demonstrates tens of microns precision.
\end{abstract}

Keywords: Optical correlator, Fourth order interferometer, Intensity interferometer, Laser ranging, Optical frequency combs, Fiber lasers, Photon number detectors

\section{INTRODUCTION}

Precise distance/time or pulse width measurements using optical signals are a well-established discipline. New technologies and methods have been historically developed to increase the precision and accuracy of the distance/time or pulse width measurements. A driving engineering factor is also the receiver sensitivity and laser transmitter and receiver wavelength compatibility. For lower precision measurements, a direct detection receiver is employed with a single high bandwidth photodetector. Optical direct detection receiver bandwidths in excess of $100 \mathrm{GHz}$ have been achieved. For high sensitivity, single photon sensitive detectors have been employed in direct detection schemes. Coherent (e.g. heterodyne or homodyne) detection has been employed where a local oscillator signal is multiplied by the received signal in a coherent optical mixing scheme to improve the receiver sensitivity. Since two optical signals are mixed directly, the coherent receiver product term is sometimes denoted $2^{\text {nd }}$ order optical interference. For optical signals with bandwidths that exceed the direct photodetector bandwidth a space-time optical auto-correlator is used. The optical auto-correlator incorporates a nonlinear optical crystal to directly form the product between the optical signal and a delayed copy of the optical signal. Since distance and time are related by the velocity of light in this scheme the pulse width can be measured by changing the distance in one of the arms of the optical correlator and directly relating this to time delay. This type of optical autocorrelator can be used to measure the pulse width of very short optical pulses (femtoseconds). One purpose of our new innovation described here is to introduce a new correlation method that does not require the nonlinear optical crystal for the mixing (multiplying) of the optical signal. The new method described here relies on the fourth order interference. It therefore can be used on much weaker optical signals than have been traditionally used in the nonlinear optical crystal correlator.

In addition, a cross-correlation can be formed between two beams of light: a weak (i.e. low intensity) "signal" beam of photons and a much stronger (higher intensity) "local oscillator" beam of photons. The fourth-order interference product term then allows the detection of very weak signals in exact analogy to a heterodyne receiver (first introduced by Edwin Armstrong $^{1}$ ) in the radio frequency (RF) domain and coherent receivers in the optical domain. A key difference between a correlation receiver and a coherent receiver is that the correlation receiver relies on computing time-averaged statistics of the optical signals whereas the coherent receiver can operate on the instantaneous signal.

Although the correlation receiver can be used with many optical sources, the invention of optical frequency combs provides a unique optical transmitter source because the optical frequency comb (i.e. a mode-locked laser that is frequencylocked to an atomic transition) provides a wide-sense cyclostationary process that is advantageous for the optimizing signal recovery using correlation receivers. 


\section{FOURTH-ORDER OPTICAL CORRELATOR}

Over the last few decades, a higher order optical correlation has been studied from "two-photon" optical sources that is known as fourth order optical interference ${ }^{2}$. The fourth-order interference was first discovered in $1956^{3}$. The physics underlying the device described here relies on this fourth order interference. One method to observe the fourth order interference is to delay one signal from a $2^{\text {nd }}$ signal using one arm of the optical auto-correlator. The optical correlator is formed with a beam splitter and two detectors and a means of delaying one arm of the path between the beam splitter and a detector. The optical correlator uses a low-noise detector that is fast enough to resolve each pulse of photons, but can be slow enough to time integrate the photons in each pulse or it can be a detector that can resolve photon number (intensity). A key part of the invention is in the processing of the electrical signals from the detector(s). Two optical signals (A and B) are directed to a beam splitter where they impinge on two detectors (D1 and D2). The heart of the invention is to form the correlation product integral kernel. One method to form the product is to calculate the variance of the photon number (intensity) (N1 and N2) of the difference of the optical signals on the two detectors. Figure 1 shows the optical correlation receiver diagram.

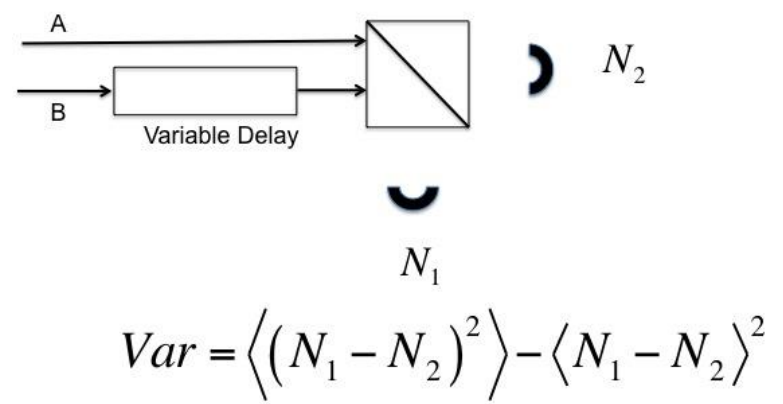

Fig. 1 The variance is calculated at each step of a variable delay line.

A typical embodiment of the optical correlation receiver is shown in Figure 2.

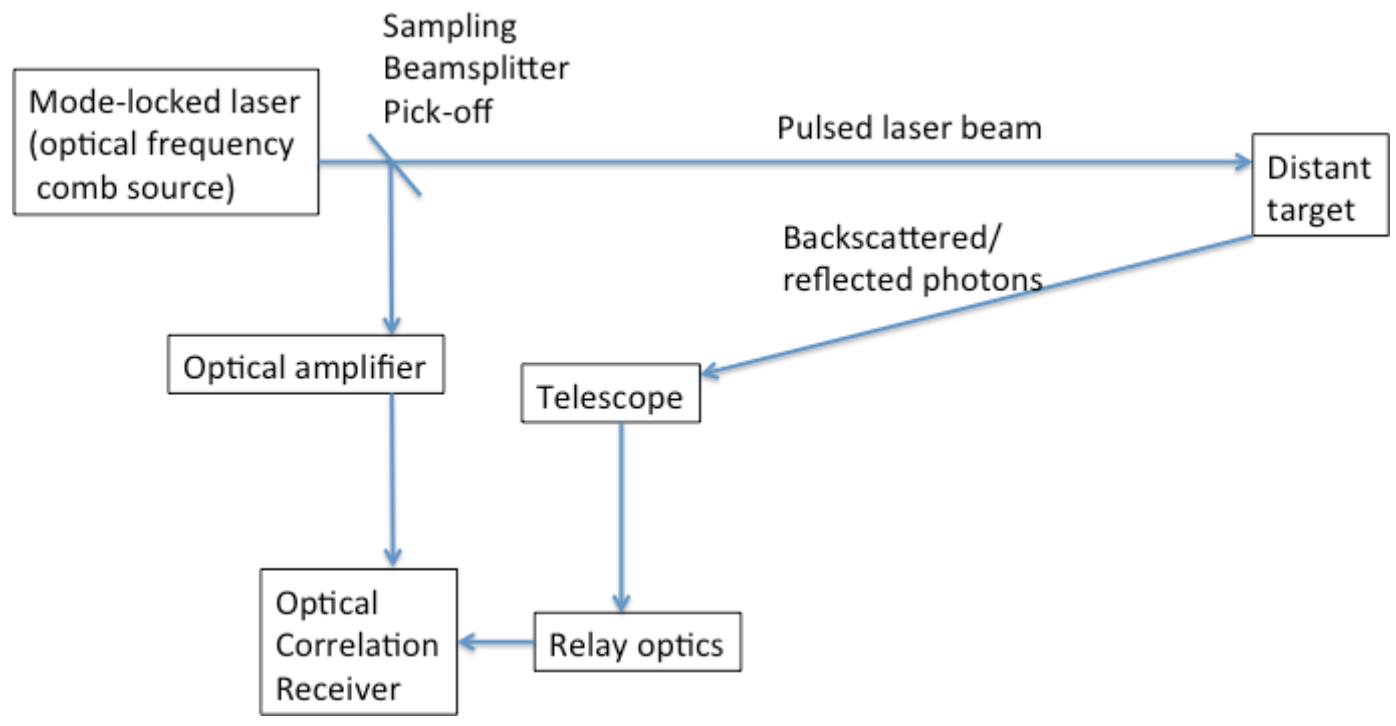

Fig. 2 One example of use of the optical correlation receiver

A mode-locked laser - ideally an optical frequency comb (i.e. the laser is frequency locked to an external reference - an atomic transition) - produces a pulsed optical output with a well-defined time spacing between pulses. A small portion of the outgoing pulse train (a replica of the optical signal) is sent to an optical amplifier. The outgoing pulse train is sent to a distant target where some portion of it is backscattered and/or reflected. A small portion of the backscattered photons 
are collected with an optical telescope. The photons collected by the telescope are sent through some relay optics to the input of the optical correlation receiver (described below).

A diagram of the optical correlation receiver is shown in Figure 3.

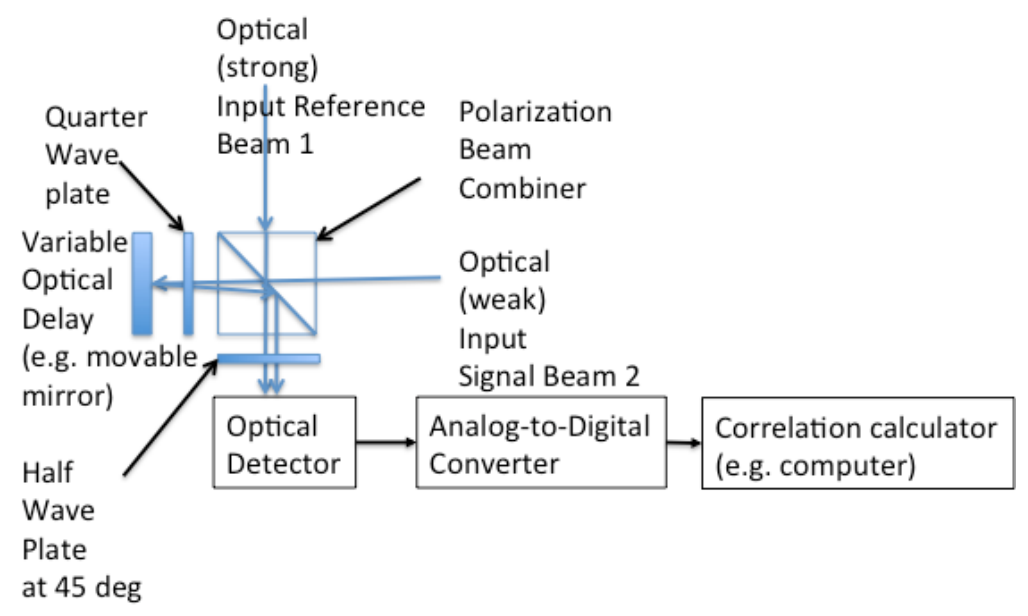

Fig. 3 Example of Optical Correlation Receiver using a moveable mirror for optical delay.

The optical correlation receiver consists of polarization beam combiner, a variable optical delay, an optical detector or detectors, an analog-to-digital converter and a correlation calculator such as a computer or a Field Programmable Gate Array (FPGA). A strong optical beam is incident on a polarization beam combiner such that its straight-through transmission is optimized. The weak optical beam is incident on the $2^{\text {nd }}$ port of the beam combiner also such that its straight through transmission is optimized. After reflection from a moveable mirror that permits variable optical delay between the two optical beams, the polarization of the weak beam is adjusted with a quarter-wave plate to maximize its reflection from the beam combiner toward the optical detector. The configuration is such that the two beams have orthogonal polarization when reaching the detector such that the two intensities are summed on the detector and no $2^{\text {nd }}$ order interference occurs. The electrical output of the optical detector is approximately the sum of the strong and weak beam individual intensities. This sum is digitized by an analog-to-digital converter and a pulse train of the integrated intensity (i.e. the approximate photon number) of each pulse is sent to the correlation calculator. The correlation calculator then calculates the integral of the product of the two sets of pulse train intensities at a discrete set of optical delays (i.e. the cross-correlation) to give the mathematical correlation of the sum of the two intensity pulse trains. From the position of the correlation peak (i.e. the knowledge of the delay where the two beams overlap), the distance to the target can be calculated with very high precision.

\section{FOURTH-ORDER CORRELATOR THEORY}

We define a comb functioned as:

$$
\operatorname{Comb}(t)=\operatorname{comb}(t) * P u l s e(t)
$$

where $\operatorname{comb}(t)$ is the repetitive Dirac delta comb function, Pulse $(t)$ is the laser pulse shape and $*$ denotes convolution. For simplicity sake, we assume perfect 50:50 beam splitters that give equal amplitudes. For simplicity here, the optical carrier is implicit. Iskhakov gives a more complete version of the theory including the optical carrier ${ }^{4}$.

$$
\begin{aligned}
& E_{1}(t)=A(t \quad) \operatorname{Comb}(t \quad)+B(t) \operatorname{Comb}(t \quad) e^{i} \\
& E_{2}(t)=A(t \quad) \operatorname{Comb}(t \quad) \quad B(t) \operatorname{Comb}(t \quad) e^{i}
\end{aligned}
$$




$$
\begin{aligned}
& I_{1}(t)=|A(t \quad) \operatorname{Comb}(t \quad)|^{2}+|B(t) \operatorname{Comb}(t)|^{2}+A(t \quad) \operatorname{Comb}(t \quad) B(t) \operatorname{Comb}(t \quad) e^{i}+c . c . \\
& I_{2}(t)=|A(t \quad) \operatorname{Comb}(t \quad)|^{2}+|B(t) \operatorname{Comb}(t)|^{2} \quad A(t \quad) \operatorname{Comb}(t \quad) B(t) \operatorname{Comb}(t \quad) e^{i} \quad \text { c.c. }
\end{aligned}
$$

The average in the difference of the two detectors' (intensity) photon number $\left(N_{-}(\tau)\right)$ is:

$$
N()=\left(I_{1}(t) \quad I_{2}(t)\right) d t=d t\left(2 A^{*}(t \quad) \operatorname{Comb}^{*}(t \quad) B(t) \operatorname{Comb}(t) e^{i}+\text { c.c. }\right)
$$

Since the beams have equal intensity, the mean of $I_{1}(t) \quad I_{2}(t)$ is zero. The variance of the difference is:

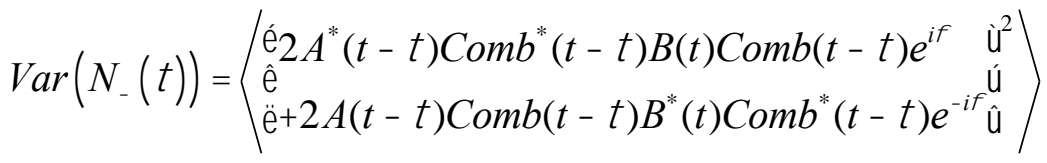

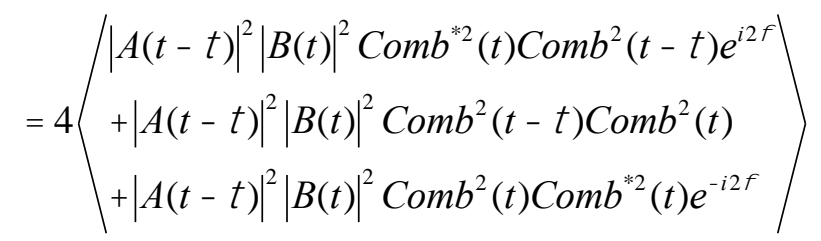

When a random phase is introduced in one beam (e.g. with a moving mirror), the terms containing $\phi$ in the exponent average to zero. The variance of the difference is given by:

$$
\operatorname{Var}(N())=4 \quad|A(t \quad)|^{2}|B(t)|^{2} \operatorname{Comb}^{2}(t \quad) \operatorname{Comb}^{2}(t) d t
$$

This is the correlation function.

We constructed a MathCad simulation to show the utility of calculating the correlation function from the photon number variance difference for the case of Poisson intensity distributed pulse trains.

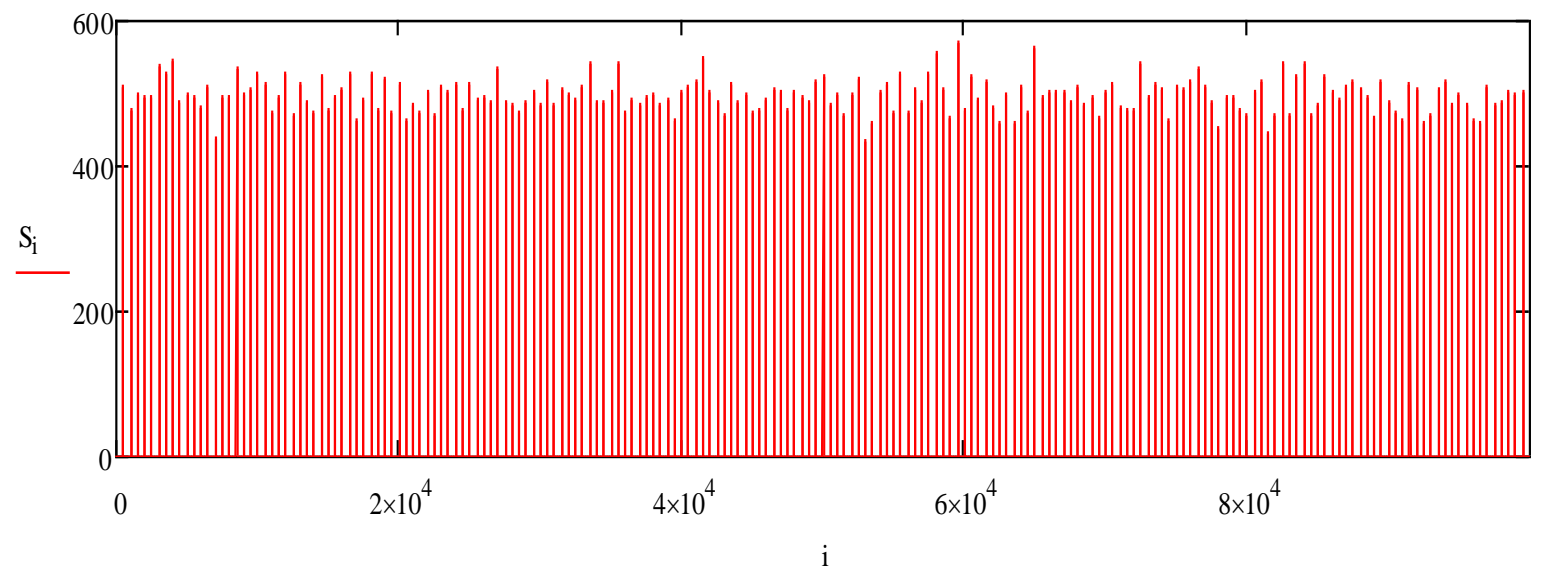

Figure 4. Example comb pulse train with Poisson distributed photon number intensity

An example comb pulse train Figure 4 with pulse-to-pulse Poisson distributed intensity and the resulting correlation function is shown from a MathCad simulation in Figure 5. 


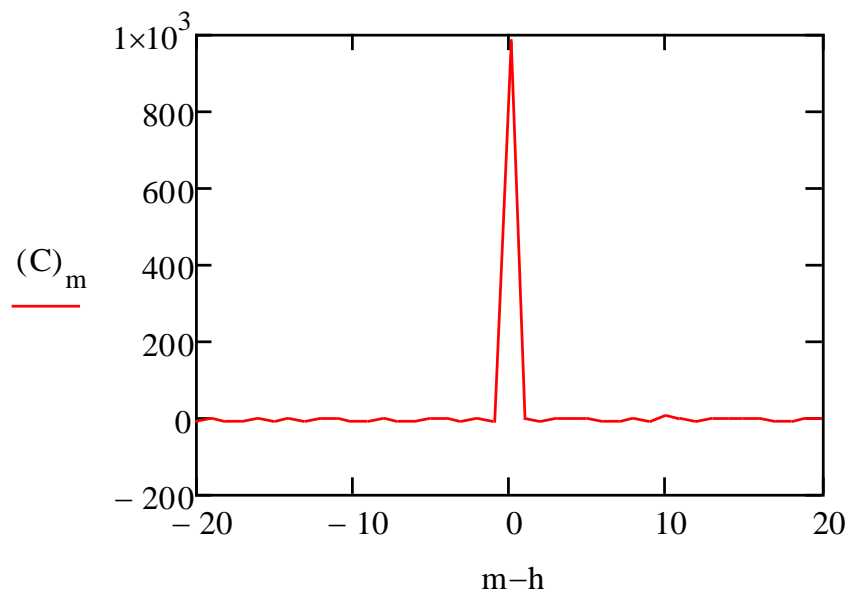

Figure 5. Optical correlator MathCad simulation output for dual detector correlation (Variance of the difference) $(\mathrm{m}-\mathrm{h}=$ delay in single comb pulse width units)

When the optical carrier is included the correlation function is multiplied by the coherence function. Iskhakov ${ }^{4}$ shows that for the case of a Gaussian pulse with the FWHM T and the coherence time $\tau_{\mathfrak{c}}$, the shape of the correlation peak is Gaussian and given by:

$$
\frac{\operatorname{Var}(N())}{\left\langle N_{+}\right\rangle}=\frac{1}{2}\left\langle N_{+}\right\rangle \exp \left[2 \ln 2 \frac{2}{2}\right]
$$

where $1 / \Delta \tau^{2}=1 / \tau_{\square}^{2}+1 / \mathrm{T}^{2}$ and $\left\langle N_{+}\right\rangle=\left\langle N_{1}+N_{2}\right\rangle$ is the sum of the mean photon numbers from the two detectors.

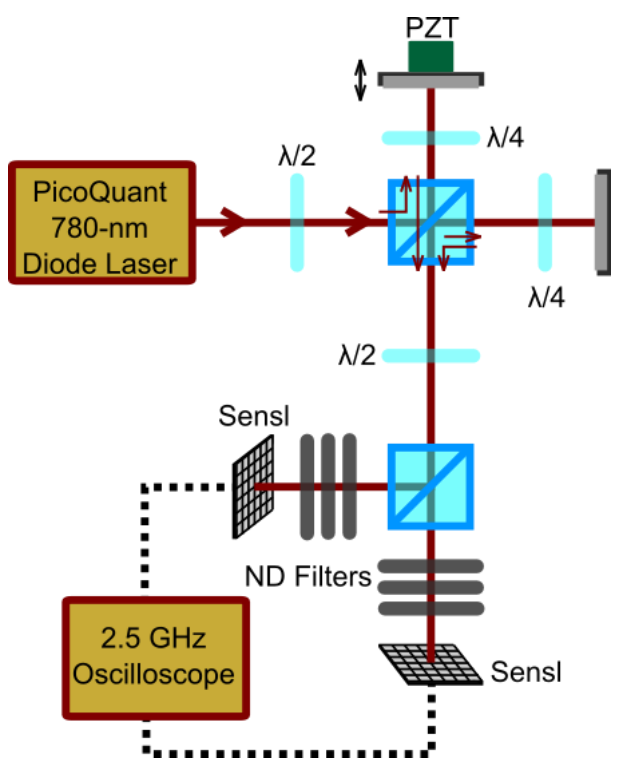

Figure 6. Fourth-order correlator proof-of-concept experiment 


\section{PROOF-OF-CONCEPT EXPERIMENTS}

For a proof-of-concept experiment we constructed a fourth-order interferometer using polarization beam splitters and quarter-wave plates as shown in Figure 6. The pulsed light source is a Picoquant $780 \mathrm{~nm}$ wavelength laser diode with a $70 \mathrm{ps}$ pulse width and $2.5 \mathrm{~nm}$ spectral width (360 fs coherence time). The detectors are silicon avalanche photodiode arrays (Sensl Model MicroFC-SMA-10010) with an on-chip high-pass filtered output that provides good photon number resolution. A random phase is introduced in one beam (e.g. with a moving mirror driven by a piezo-electric-transducer (PZT). We used an oscilloscope to subtract the outputs of the two detector arrays (directly measuring $I_{1}(t)-I_{2}(t)$ ). We measured the variance of the difference of detector outputs over 10,000 optical pulses at each micrometer position to ensure the average phase induced by the PZT was 0 . The experimentally observed fourth-order correlation peak and the theory from Equation (9) is shown in Figure 7.

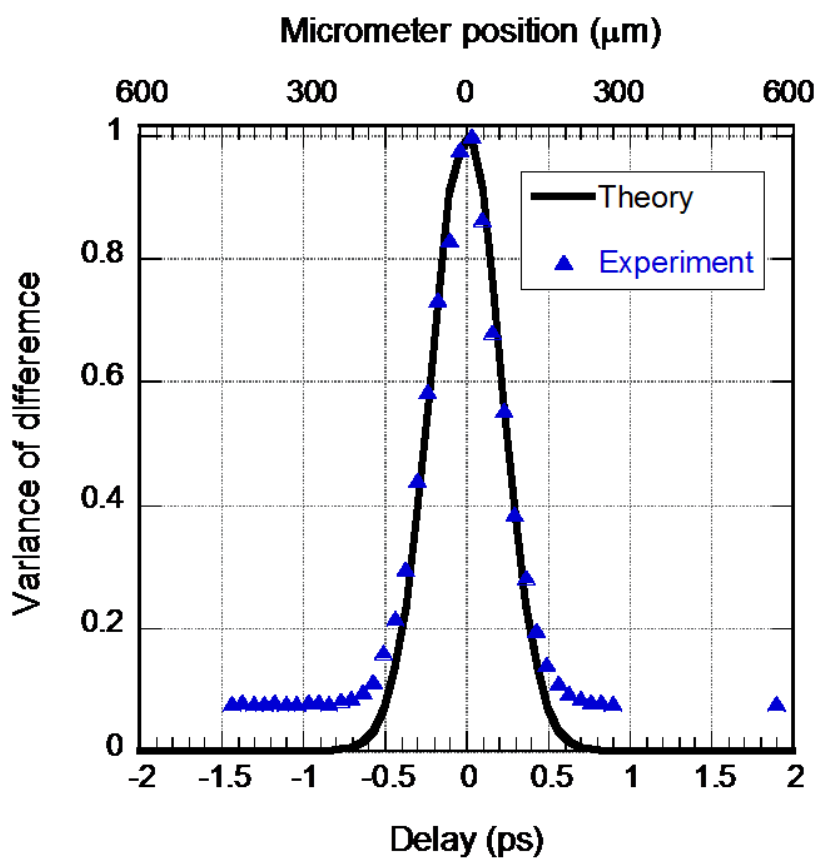

Figure 7. Normalized fourth-order correlator experimental and theoretical result

\section{SUMMARY}

We presented an optical correlation receiver that provides ultra-precise distance and/or time/pulsewidth measurements even for weak (single photons) and short (femtosecond) optical signals. This new type of optical correlation receiver uses a fourth-order (intensity) interferometer to provide micron distance measurements even for weak (single photons) and short (femtosecond) optical signals. Our preliminary proof-of principle data (using a short-pulse diode laser transmitter) demonstrates tens of microns precision. New mode-locked lasers with graphene ${ }^{5}$ and carbon nanotube ${ }^{6}$ saturable absorbers provide low-cost and robust femtosecond pulsed transmitters for laser ranging. In addition, large, commercial, low-cost Geiger-mode silicon avalanche photodiode arrays with on-chip high-pass filtered output provide high-count-rates (hundreds of Megacounts per second) with good photon number resolution. These technologies offer great promise for ground and space-based laser ranging systems.

\section{REFERENCES}

[1] Armstrong, EH "The super-heterodyne - Its origin, development, and some recent improvements," Proceedings of the Institute of Radio Engineers Vol. 12, No. 5, 539-552 (1924) 
[2] Mandel, L. "Quantum effects in one-photon and two-photon interference," Reviews of Modern Physics Vol. 71, No. 2, 274-282 (1999)

[3] Hanbury-Brown, R., Twiss, R. "Correlation between photons in two coherent beams of light," Nature Vol. 177, 1325 (1956)

[4] Iskhakov, T. Sh; Spasibko, K. Yu; Chekhova, M. V.; et al. "Macroscopic Hong-Ou-Mandel interference," New Journal of Physics Vol. 15, 093036 (2013)

[5] Martinez, Amos; Yamashita, Shinji "10 GHz fundamental mode fiber laser using a graphene saturable absorber," Applied Physics Letters Vol. 101, No. 4, 041118 (2012)

[6] Yu, Zhenhua; Wang, Yonggang; Zhang, Xiao; et al. "A 66 fs highly stable single wall carbon nanotube mode locked fiber laser," Laser Physics Vol. 24, No. 1, 015105 (2014) 